This item was submitted to Loughborough's Research Repository by the author.

Items in Figshare are protected by copyright, with all rights reserved, unless otherwise indicated.

\title{
Dynamic resource allocation for MC-NOMA VWNs with imperfect SIC
}

PLEASE CITE THE PUBLISHED VERSION

https://doi.org/10.1109/PIMRC.2017.8292371

PUBLISHER

(c) IEEE

VERSION

AM (Accepted Manuscript)

LICENCE

CC BY-NC-ND 4.0

REPOSITORY RECORD

Tweed, Daniel, Saeedeh Parsaeefard, Mahsa Derakhshani, and Tho Le-Ngoc. 2019. "Dynamic Resource Allocation for MC-NOMA Vwns with Imperfect SIC". figshare. https://hdl.handle.net/2134/26472. 


\title{
Dynamic Resource Allocation for MC-NOMA VWNs with Imperfect SIC
}

\author{
Daniel Tweed*, Saeedeh Parsaeefard ${ }^{\dagger}$, Mahsa Derakhshani ${ }^{\ddagger}$, Tho Le-Ngoc* \\ * Department of Electrical \& Computer Engineering, McGill University, Montreal, Canada \\ $\dagger$ Iran Telecommunications Research Center, Tehran, Iran \\ $\ddagger$ Wolfson School of Mechanical, Electrical and Manufacturing Engineering, Loughborough University, UK \\ E-mail: daniel.tweed@mail.mcgill.ca; s.parsaeifard@itrc.ac.ir; m.derakhshani@lboro.ac.uk; tho.le-ngoc@mcgill.ca
}

\begin{abstract}
In this work, we investigate the uplink resource allocation problem for virtualized wireless networks (VWNs) supported by multi-carrier non-orthogonal multiple access (MC-NOMA) and present a sensitivity analysis of such a system to imperfect successive interference cancellation (SIC) and various system parameters. The proposed algorithm for power and sub-carrier allocation is derived from the non-convex optimization minimizing power subject to rate and sub-carrier reservations, for which an optimal solution is NP-hard. To develop an efficient $s$ olution, $w$ e d ecompose $t$ he o ptimization into separate power and sub-carrier allocation problems and propose an iterative algorithm based on successive convex approximation and complementary geometric programming. Simulation results demonstrate that compared to orthogonal multiple access, for imperfect SIC with residual interference even up to $10 \%$, the proposed algorithm for MC-NOMA can offer significant improvement in spectrum and power efficiency.
\end{abstract}

Index Terms-Wireless virtualization, MC-NOMA, resource allocation, complementary geometric programming.

\section{INTRODUCTION}

Multi-carrier non-orthogonal multiple access (MCNOMA) and virtualized wireless networks (VWNs) are being positioned as promising techniques to meet the targets set for fifth $\mathrm{g}$ eneration $\mathrm{n}$ etworks a nd beyond. This work investigates MC-NOMA supporting VWN slices representing classes of devices which have stringent power limitations and strict quality of service (QoS) requirements. Due to these limitations, and the greater resources for performing successive interference cancellation (SIC) at the base station (BS), we limit our investigations to the uplink (UL) case and allow higher order multiplexing and optimize over both subcarrier and power allocation. The joint resource allocation problem is formulated as minimizing user equipment (UE) transmit power under slice and network constraints. Complementary geometric programming (CGP) and variable relaxation are applied to the resulting nonconvex NP-hard optimization to develop an efficient iterative algorithm for sub-carrier and power allocation. We examine the effects of SIC imperfections on UL MC-NOMA based VWN with rate- and resource-based QoS over several system parameters. Simulation results verify the proposed algorithm for VWN provides better performance than OMA in terms of UE power levels and traffic density, even in the presence of high interuser interference from imperfect SIC.

The remainder of this paper is organized as follows: Section II presents the system model and problem formulation. The derivation of the proposed algorithm is provided in Section III. Section IV presents simulation results and analysis, followed by the conclusion in Section V.

\section{System Model}

Consider a single BS serving a set of slices, $\mathcal{S}$, in which each slice $s \in \mathcal{S}$ has its own set of users, $\mathcal{K}_{s}=\left\{1, \ldots, K_{s}\right\}$, comprised of classes of devices with distinct traffic patterns, priorities, and service requirements. To meet these needs, each slice has negotiated QoS as a minimum reserved slice rate, $R_{s}^{\text {rsv }}$, and a minimum reserved number of sub-carriers, $N_{s}^{\text {rsv }}$, allocated to the slice. The total number of users in the system is $K=\sum_{s \in \mathcal{S}} K_{s}$ and each of the available sub-carriers, $n \in \mathcal{N}=\{1, \ldots, N\}$, can be shared by at most $N_{\max }$ users. A Rayleigh fading model is assumed, and the channel gain between user $k_{s}$ and the BS on sub-carrier $n$ is given by

$$
h_{k_{s}, n}=\chi_{k_{s}, n} d_{k_{s}}^{-\lambda} \text {, }
$$

where $\chi_{k_{s}, n} \sim \operatorname{Exp}(1)$ is the channel coefficient; $d_{k_{s}}$ is the distance between user $k_{s}$ and the BS; and $\lambda$ is the path-loss exponent.

In each sub-carrier $n$, for UL MC-NOMA, users are ranked according to channel gain such that $h_{1, n}>$ $h_{2, n}>\cdots>h_{K, n}$ [1]. Then, to decode user ranked $i$, the BS performs SIC to remove the signals from all users whose indices are lower than $i$. The remaining users, whose indices are greater than $i$, are treated as unresolvable interference. Thus, assuming user $i$ transmits with power $\beta_{i, n}$, the signal-to-interference-plusnoise ratio (SINR) after SIC at the BS is

$$
\gamma_{i, n}=\frac{\beta_{i, n} h_{i, n}}{\sigma_{i, n}^{2}+I_{i, n}^{r}+I_{i, n}^{e}},
$$

where $\sigma_{i, n}^{2}$ is the noise power, $I_{i, n}^{r}=\sum_{j=i+1}^{K} \beta_{j, n} h_{j, n}$ is the residual interference from users $i<j \leq K$, and $I_{i, n}^{e}=\sum_{j=1}^{i-1} \beta_{j, n} h_{j, n}\left|x_{j}-\hat{x}_{j}\right|^{2}$ is the interference resulting from imperfect cancellation of users $1 \leq j<i$. Here $x_{j}-\hat{x}_{j}$ is the difference between the actual and estimated signal transmitted by user $j$. For perfect SIC, $\hat{x}_{j}=x_{j}$ and then $I_{i, n}^{e}=0$. Otherwise, some portion 


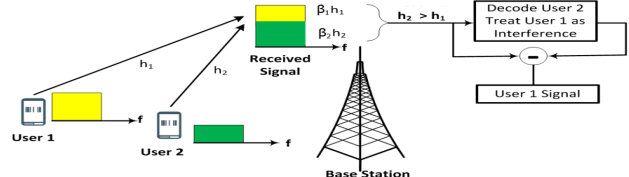

Fig. 1. Illustration of an UL MC-NOMA system with two users. Power is allocated to maintain distinctness of individual signals in the superposed received signal. User 2 is the stronger received component so the BS decodes User 2 then subtracts the result from the received signal to resolve User 1.

of the received power, $\beta_{j, n} h_{j, n}$, remains as interference. The magnitude of SIC error is dependent on the type of SIC employed, the number of user signals being cancelled, and channel and user mobility conditions. To account for this, we define the residual interference power due to the combined SIC error sources as $\beta_{j, n} h_{j, n} \sigma_{e}^{2}$, where $\sigma_{e}^{2}=\mathbf{E}\left[\left|x_{j}-\hat{x}_{j}\right|^{2}\right]$ is the expected level of cancellation, e.g., $\sigma_{e}^{2}=0.01$ equates to $20 \mathrm{~dB}$ cancellation [2].

The rate for user ranked $i$ on sub-carrier $n$ is then

$$
R_{i, n}=\log _{2}\left(1+\gamma_{i, n}\right) \text {. }
$$

Defining $\alpha_{k_{s}, n} \in\{0,1\}$ to be the sub-carrier allocation indicator, where $\alpha_{k_{s}, n}=1$ means user $k_{s}$ is allocated to sub-carrier $n$, the sum rate achieved by each slice $s$ can be expressed as

$$
R_{s}=\sum_{k_{s} \in \mathcal{K}_{s}} \sum_{n \in \mathcal{N}} \alpha_{k_{s}, n} R_{i, n}
$$

Considering the sub-carrier allocation, $\boldsymbol{\alpha}=\left[\alpha_{k_{s}, n}\right]$ and power allocation $\boldsymbol{\beta}=\left[\beta_{k_{s}, n}\right]$, we seek to minimize transmit power for user while meeting QoS requirements, which can be expressed as

$$
\begin{aligned}
& \mathrm{C} 1: R_{s} \geq R_{s}^{\mathrm{rsv}}, \forall s \in \mathcal{S}, \\
& \mathrm{C} 2: \sum_{n \in \mathcal{N}} \sum_{k_{s} \in \mathcal{K}_{s}} \alpha_{k_{s}, n} \geq N_{s}^{\mathrm{rsv}}, \forall s \in \mathcal{S} .
\end{aligned}
$$

Power must also be maintained below the maximum allowable UE transmit power, which can be expressed

$$
\text { C3 : } \sum_{n \in \mathcal{N}} \beta_{k_{s}, n} \leq P_{\max }, \forall s \in \mathcal{S}, \forall k_{s} \in \mathcal{K}_{s} .
$$

To limit the complexity of performing SIC at the BS we have another constraint on $\alpha_{k_{s}, n}$ as

$$
\text { C4 : } \sum_{s \in \mathcal{S}} \sum_{k_{s} \in \mathcal{K}_{s}} \alpha_{k_{s}, n} \leq N_{\max }, \forall n \in \mathcal{N} .
$$

Finally, to ensure that power is not allocated to subcarriers which the UE is not using we have the following constraint

$$
\begin{aligned}
\text { C5 : } \beta_{k_{s}, n}-\alpha_{k_{s}, n} \times & P_{\max } \leq 0, \\
& \forall n \in \mathcal{N}, \forall s \in \mathcal{S}, \forall k_{s} \in \mathcal{K}_{s} .
\end{aligned}
$$

Then the optimization to minimize required UE transmit power can be expressed as

$$
\min _{\boldsymbol{\alpha}, \boldsymbol{\beta}} \max _{\substack{\forall \\ \forall k_{s} \in \mathcal{K}}} \sum_{n \in \mathcal{N}} \alpha_{k_{s}, n} \beta_{k_{s}, n}, \text { subject to: } \mathrm{C} 1-5 .
$$

III. Proposed Algorithm

The constraint given by $\mathrm{C} 1$ is non-convex due to the interference terms in (3), which relies on both power and sub-carrier allocation. Further, C2 and C5 are non-

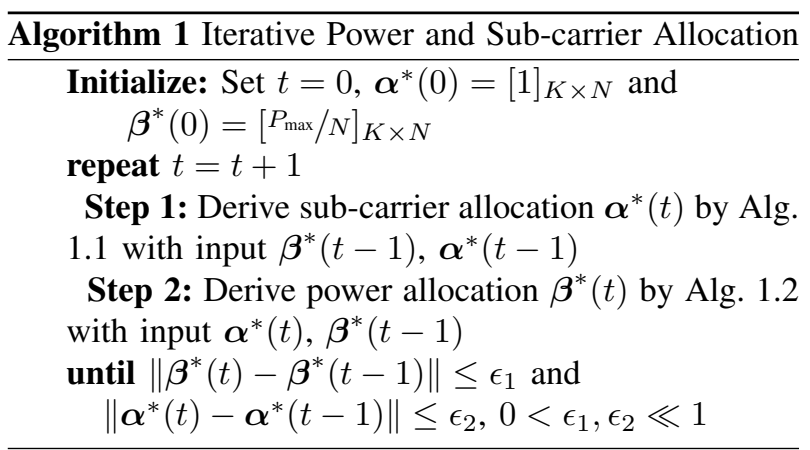

convex and the complexity induced by the binary variable yields a problem which also NP-hard. To efficiently solve the problem in (5) the iterative algorithm shown in Algorithm 1 based on successive convex approximation (SCA) is proposed.

Initially, power is evenly distributed across all subcarriers and in Step 1 an optimal sub-carrier allocation is derived. In Step 2 an optimal power allocation is derived for the sub-carrier allocation found in Step 1. These steps are repeated until the solution to each sub-problem is not much different than the previous iteration. While the sub-problems are simpler than (5), they each remain challenging and an iterative approach is again taken. We apply variable relaxation to reduce the complexity of the problem and CGP to convert each into the geometric programming (GP) form and approximate non-convex constraints using the arithmetic-geometric mean approximation (AGMA) on each iteration until the solution converges. The solution to the relaxed problem provides a lower bound on the minimization and the output of Alg. 1 can be recovered to binary via integer rounding to produce a feasible solution to the original problem.

Interested readers on CGP and AGMA are referred to [3]-[5]. The convergence of CGP has been proven in [3] and it has been shown that the output of algorithms based on SCA and AGMA converges to a local optimum of the original problem and have very close performance to the optimal solution [4], [5].

\section{A. Sub-carrier Allocation}

Given a fixed power allocation, we have the following optimization problem

$$
\min _{\boldsymbol{\alpha}} \max _{\substack{\forall s \in \mathcal{S} \\ \forall k_{s} \in \mathcal{K}_{s}}} \sum_{n \in \mathcal{N}} \alpha_{k_{s}, n} \beta_{k_{s}, n}, \text { subject to: C1, C2, C4. }
$$

Each of $\mathrm{C} 1$ and $\mathrm{C} 2$ is non-convex and, due to the binary variable $\boldsymbol{\alpha}$, this problem suffers from high computational complexity. First we reduce the complexity by relaxing $\alpha_{k} \in[0,1]$. For each iteration, $t_{1}$, we can then approximate the non-convex constraints as convex functions by applying AGMA. C1 can be written as

$$
\frac{R_{s}^{\mathrm{rsv}}}{\sum_{k_{s} \in \mathcal{K}_{s}} \sum_{n \in \mathcal{N}} R_{k_{s}, n}} \leq 1
$$




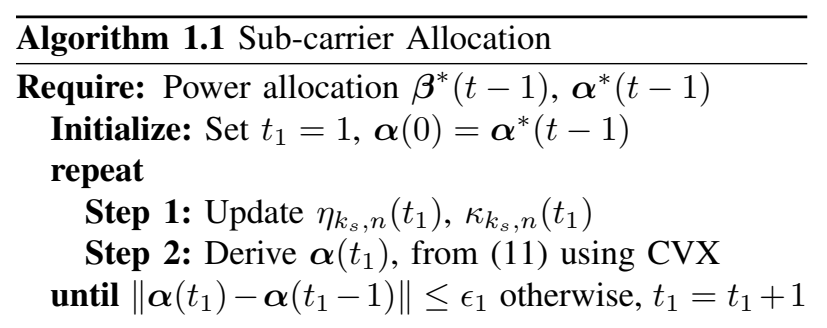

the left-hand side of which can be approximated by the following convex function

$$
x_{s}\left(t_{1}\right)=R_{s}^{\mathrm{rsv}} \times \prod_{k_{s} \in \mathcal{K}_{S}} \prod_{n \in \mathcal{N}}\left(\frac{\alpha_{k_{s}, n}\left(t_{1}\right) R_{k_{s}, n}}{\eta_{k_{s}, n}\left(t_{1}\right)}\right)^{-\eta_{k_{s}, n}\left(t_{1}\right)} .
$$

where $\eta_{k_{s}, n}\left(t_{1}\right)=\frac{\alpha_{k_{s}, n}\left(t_{1}-1\right) R_{k_{s}, n}}{\sum_{k_{s} \in \mathcal{K}_{s}} \sum_{n \in \mathcal{N}} \alpha_{k_{s}, n}\left(t_{1}-1\right) R_{k_{s}, n}}$

Similarly, we can transform C2 and define the convex function

$$
y_{s}\left(t_{1}\right)=N_{s}^{\mathrm{rsv}} \times \prod_{k_{s} \in \mathcal{K}_{S}} \prod_{n \in \mathcal{N}}\left(\frac{\alpha_{k_{s}, n}\left(t_{1}\right)}{\kappa_{k_{s}, n}\left(t_{1}\right)}\right)^{-\kappa_{k_{s}, n}\left(t_{1}\right)},
$$

where $\kappa_{k_{s}, n}\left(t_{1}\right)=\frac{\alpha_{k_{s}, n}\left(t_{1}-1\right)}{\sum_{k_{s} \in \mathcal{K}_{s}} \sum_{n \in \mathcal{N}} \alpha_{k_{s}, n}\left(t_{1}-1\right)}$. Thus, at each iteration, $t_{1}$, we solve

$$
\begin{aligned}
& \min _{\boldsymbol{\alpha}} \max _{\substack{\forall s \in \mathcal{S} \\
\forall k_{s} \in \mathcal{K}_{s}}} \sum_{n \in \mathcal{N}} \alpha_{k_{s}, n}\left(t_{1}\right) \beta_{k_{s}, n} \\
& \text { Subject to: C4, } x_{s}\left(t_{1}\right) \leq 1, y_{s}\left(t_{1}\right) \leq 1, \forall s \in \mathcal{S} .
\end{aligned}
$$

as described in Algorithm 1.1.

B. Power Allocation

Given a sub-carrier allocation, we solve the following optimization problem

$$
\min _{\boldsymbol{\beta}} \max _{\substack{\forall s \in \mathcal{S} \\ \forall k_{s} \in \mathcal{K}_{s}}} \sum_{n \in \mathcal{N}} \alpha_{k_{s}, n} \beta_{k_{s}, n}, \text { subject to : C1, C3, C5. }
$$

Here, $\mathrm{C} 1$ is non-convex due to the interference terms in the expression for $R_{k_{s}, n}$. If we rewrite the rate expression and remove the logarithm, $\mathrm{C} 1$ can be expressed as

$$
\begin{gathered}
\prod_{k_{s} \in \mathcal{K}_{S}} \prod_{n \in \mathcal{N}}\left(\frac{\sigma_{i, n}^{2}+I_{i, n}^{r}+I_{i, n}^{e}}{\sigma_{i, n}^{2}+I_{i, n}^{r}+I_{i, n}^{e}+\beta_{i, n} h_{i, n}}\right)^{\alpha_{k_{s}, n}} \leq 2^{-R_{s}^{\mathrm{rsv}}} \\
, \forall s \in \mathcal{S} .
\end{gathered}
$$

Substituting from the definitions of $I_{i, n}^{r}$ and $I_{i, n}^{e}$ and applying AGMA, at iteration $t_{2}$ we can then approximate the product terms in (13) with the following convex function

$$
\begin{aligned}
& x_{i, n}\left(t_{2}\right)=\left(\sigma_{i, n}^{2}+I_{i, n}^{r}\left(t_{2}\right)+I_{i, n}^{e}\left(t_{2}\right)\right) \times \\
& \left(\frac{\sigma^{2}}{\phi_{i, n}\left(t_{2}\right)}\right)^{-\phi_{i, n}\left(t_{2}\right)} \times \prod_{j=1}^{i-1}\left(\frac{\beta_{j, n}\left(t_{2}\right) h_{j, n} \sigma_{e, j}^{2}}{\gamma_{j, n}\left(t_{2}\right)}\right)^{-\gamma_{j, n}\left(t_{2}\right)} \times \\
& \prod_{j=i+1}^{K}\left(\frac{\beta_{j, n}\left(t_{2}\right) h_{j, n}}{\mu_{j, n}\left(t_{2}\right)}\right)^{-\mu_{j, n}\left(t_{2}\right)} \times\left(\frac{\beta_{i, n}\left(t_{2}\right) h_{i, n}}{\rho_{i, n}\left(t_{2}\right)}\right)^{-\rho_{i, n}\left(t_{2}\right)},
\end{aligned}
$$

where $\phi_{i, n}\left(t_{2}\right)=\sigma^{2} / \zeta_{i, n}\left(t_{2}\right)$,

$\gamma_{j, n}\left(t_{2}\right)=\beta_{j, n}\left(t_{2}-1\right) h_{j, n} \sigma_{e, j}^{2} / \zeta_{i, n}\left(t_{2}\right)$,

$\mu_{j, n}\left(t_{2}\right)=\beta_{j, n}\left(t_{2}-1\right) h_{j, n} / \zeta_{i, n}\left(t_{2}\right)$,

$\rho_{i, n}\left(t_{2}\right)=\beta_{i, n}\left(t_{2}-1\right) h_{i, n} / \zeta_{i, n}\left(t_{2}\right)$,

$\zeta_{i, n}\left(t_{2}\right)=\sigma^{2}+I_{j, n}^{r}\left(t_{2}-1\right)+I_{j, n}^{e}\left(t_{2}-1\right)+\beta_{i, n}\left(t_{2}-1\right) h_{i, n}$.

Then at each iteration, $t_{2}$, we solve

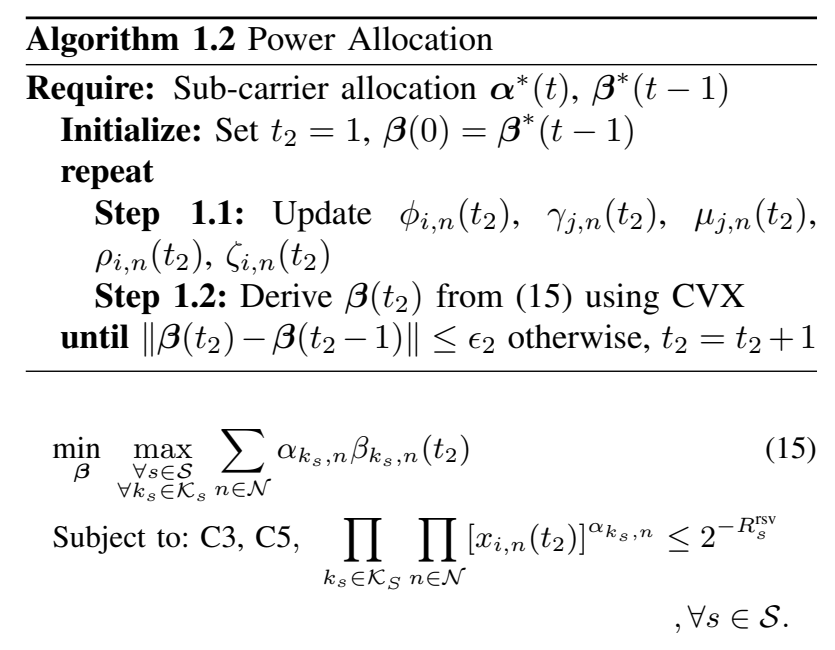

as described in Algorithm 1.2.

\section{Computational Complexity}

When CVX is used to solve the problems in (11) and (15) an interior point method is used. The number of required iterations to solve by this method is equal to $\frac{\log c / t^{0} \delta}{\log (\xi)}$, where $c$ is the total number of constraints, $t^{0}$ is the initial point, $0<\delta \ll 1$ is the stopping criterion, and $\xi$ is used for updating the accuracy of the method [6]. For (11), the total number of constraints is $c_{1}=2 S+N$ and for (15) we have $c_{2}=S+K+K N$. In Algorithms 1.1 and 1.2 the worst-case number of computations required to convert to the GP form using AGMA is $i_{1}=4 K N$ and $i_{2}=K^{2} N+5 K N$. Therefore, each algorithm $1 . j$ has complexity on the order

$$
i_{j} \times \log \left(c_{j} /\left(t^{0} \delta\right)\right) \log ^{-1}(\xi), j=1,2 .
$$

From (16) we see that the Alg. 1.2 has higher complexity than Alg. 1.1 and is more sensitive to $K$ and $N$. The number of iterations required to achieve convergence is studied further in Section IV.

\section{Simulation Results}

To evaluate the proposed algorithm, we simulate a single cell VWN with $N=16$ sub-carriers which can each be shared by at most $N_{\max }=4$ users, supporting two slices each with $K_{s}=4$ users, except where otherwise noted. For comparison, results for OMA are presented where we have set $N_{\max }=1$. In all trials we have $P_{\max }=23 \mathrm{dBm}$, and $R_{s}^{\mathrm{rsv}}=R^{\mathrm{rsv}}$ and $N_{s}^{\mathrm{rsv}}=N^{\mathrm{rsv}}$ for all slices. The users are placed randomly within the BS coverage area following a uniform distribution and the channels gains are derived according to the Rayleigh fading model with $\lambda=3$ and distances normalized to the radius of the coverage area. Errors in performing SIC are introduced as residual interfering signal power with $\sigma_{e}^{2} \in\{0,0.01,0.05,0.10\}$, i.e., up to $10 \%$ residual power remaining as inter-user interference after performing SIC, and $\sigma_{e}^{2}=0$ represents the perfect SIC case. The results shown are taken over the average of 100 channel realizations. 


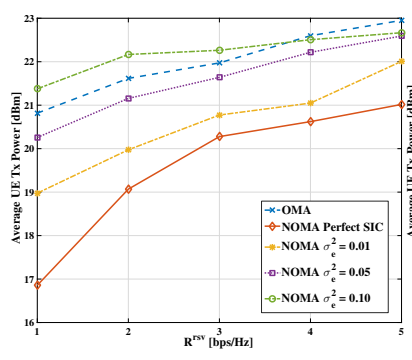

(a) Versus $R^{\mathrm{rsv}}, N^{\mathrm{rsv}}=1$

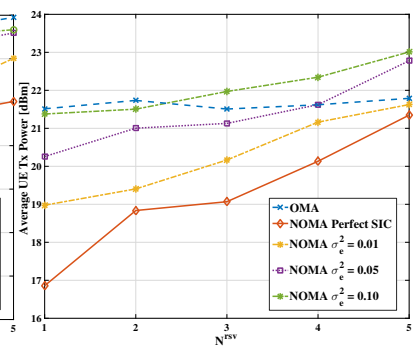

(b) Versus $N^{\mathrm{rsv}}, R^{\mathrm{rsv}}=1 \mathrm{bps} / \mathrm{Hz}$ Fig. 2. Average UE transmit power

The effect of slice reservations on transmit power are plotted in Fig. 2. Fig. 2 (a) shows the average UE transmit power per UE versus $R^{\text {rsv }}$. Power increases with $R^{\text {rsv }}$ for both OMA and MC-NOMA because each user must transmit with higher power to satisfy the slice reservation. With imperfect SIC, as expected, the required power scales with increasing SIC error due to the increased inter-user interference on shared subcarriers. However, MC-NOMA is more power-efficient than OMA for all but $\sigma_{e}^{2}=0.10$ with $R^{\text {rsv }} \leq 4 \mathrm{bps} / \mathrm{Hz}$. For example, for $R^{\mathrm{rsv}}=1 \mathrm{bps} / \mathrm{Hz}$, MC-NOMA requires an average $3.96 \mathrm{~dB}, 1.84 \mathrm{~dB}$, and $0.56 \mathrm{~dB}$ lower power than OMA for $\sigma_{e}^{2}=0$, i.e. perfect SIC, 0.01, and 0.05, respectively. For $R^{\text {rsv }}=3 \mathrm{bps} / \mathrm{Hz}$, MC-NOMA requires an average of $1.69 \mathrm{~dB}, 1.2 \mathrm{~dB}$, and $0.33 \mathrm{~dB}$ lower power than OMA for $\sigma_{e}^{2}=0,0.01$, and 0.05 , respectively.

The average UE transmit power versus $N^{\text {rsv }}$ is plotted in Fig. 2 (b). Even in the case of perfect SIC, as $N^{\text {rsv }}$ increases, required average UE transmit power increases since sub-carrier sharing is required to meet reservations and inter-user interference from higher ranked users increases. The effects of $N^{\text {rsv }}$ on OMA are not significant with only one user in each sub-carrier. In MC-NOMA, there is more flexibility in sharing stronger channels and avoiding weaker ones to minimize power but increasing $N^{\text {rsv }}$ reduces this flexibility, especially for $N^{\text {rsv }} \geq K_{s}$. For $N^{\text {rsv }}=1$, MC-NOMA requires an average of 4.65 $\mathrm{dB}, 2.53 \mathrm{~dB}$, and $1.25 \mathrm{~dB}$ lower power than OMA for $\sigma_{e}^{2}=0,0.01$, and 0.05 , respectively. However, for $N^{\text {rsv }}$ increased to 3, MC-NOMA requires an average of only $2.44 \mathrm{~dB}, 1.34 \mathrm{~dB}$, and $0.38 \mathrm{~dB}$ lower power than OMA for $\sigma_{e}^{2}=0,0.01$, and 0.05 , respectively.

The slice sub-carrier allocations are plotted in Fig. 3 , versus $R^{\text {rsv }}$ and $N^{\text {rsv }}$. In each of the sub-figures, the black line shows the maximum 8 sub-carrier allocation per slice possible under OMA, as no sharing can occur. In both cases, we see lower sub-carriers allocations in the OMA case at lower slice reservations, but from the previous figures we note the higher user transmit power is required. Up to the point where OMA approaches its maximum, depending on levels of SIC error, more subcarriers may be allocated in MC-NOMA to benefit from shared strong channels. Increasing SIC error translates into lower sub-carrier allocations as inter-user interfer-

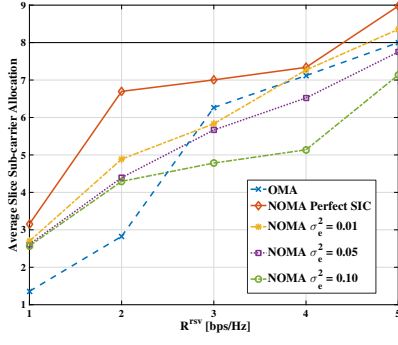

(a) Versus $R^{\mathrm{rsv}}, N^{\mathrm{rsv}}=1$

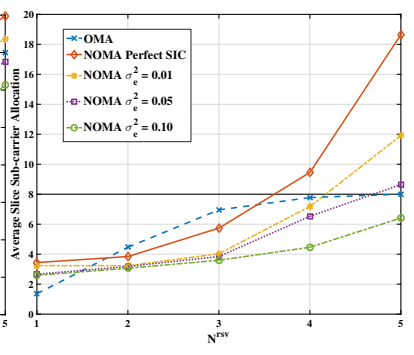

(b) Versus $N^{\mathrm{rsv}}, R^{\mathrm{rsv}}=1 \mathrm{bps} / \mathrm{Hz}$
Fig. 3. Slice average allocated sub-carriers

ence is increased. As the reservations increase, the slice sub-carrier allocations can exceed the OMA maximum. This is most clear in Fig. 3 (b), which shows allocation versus $N^{\text {rsv }}$, because the higher sub-carrier reservation for each slice must be met which reduces the flexibility of the system in avoiding weak sub-carriers.

In addition to the slice reservations, we also examined system parameters which can affect performance, the results of which are plotted in Fig. 4. Fig. 4 (a) plots the average transmit power versus the number of users $K=\sum_{s \in \mathcal{S}} \mathcal{K}_{s}$. With the number of sub-carriers fixed at $N=16$, as the number of users is increased there is a greater reliance on shared sub-carriers. In order to resolve each user signal, higher power is needed to maintain the distinctness of components in the superposed signal received at the BS to perform SIC. With increasing SIC error, weaker users in each sub-carrier experience higher inter-user interference. Although the maximum number of users per sub-carrier is fixed at $N_{\max }=4$, more sub-carriers are required to approach this value to meet rate reservations. In all cases except $\sigma_{e}^{2}=0.10$ for $K=10$, the proposed algorithm for MCNOMA outperforms the OMA case. For example, with $K=6$ MC-NOMA requires an average of $4.52 \mathrm{~dB}, 4.22$ $\mathrm{dB}, 2.98 \mathrm{~dB}$, and $1.90 \mathrm{~dB}$ lower power than OMA for $\sigma_{e}^{2}=0,0.01,0.05$, and 0.10 , respectively.

The effect of varying the total number of sub-carriers, $N$, in the system is depicted in Fig. 4 (b). We see that the required transmit power of each UE is reduced when more sub-carriers are available due to the increased flexibility in scheduling users on dedicated stronger channels. Power requirements still increase with SIC error because of the cases where sharing a strong channel is required. Fig. 4 (c) shows the sub-carrier allocations for each slice, at each level of SIC error. Slice allocations are approximately equal in all cases and we note that the sub-carriers allocated increase with the total available number of sub-carriers, but that it does not change much for the imperfect SIC cases due to the trade-off between inter-user interference and utilizing weaker channels and spreading lower power across a greater number of sub-carriers. For the perfect SIC case, where inter-user interference is only from higher ranked users, the number of allocated sub-carriers is approximately equal to the 


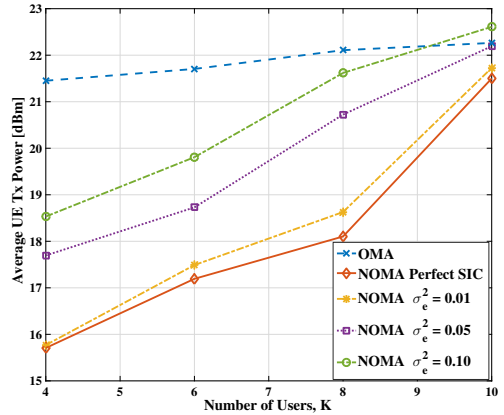

(a) UE transmit power versus $\mathrm{K}$

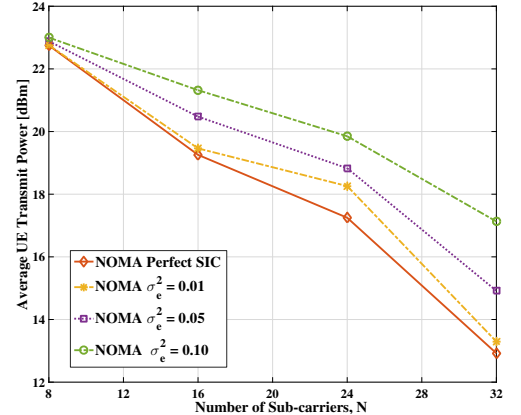

(b) UE transmit power versus $\mathrm{N}$

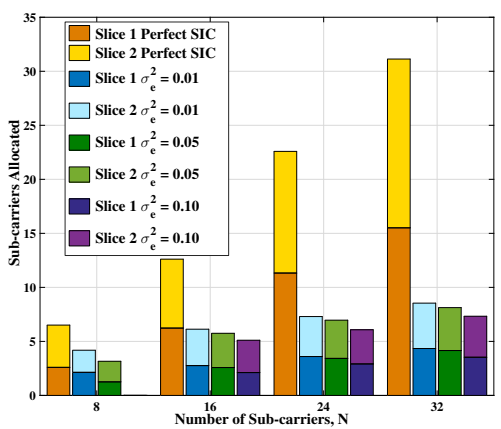

(c) Sub-carrier allocations versus $\mathrm{N}$

Fig. 4. Allocation resources versus system parameters

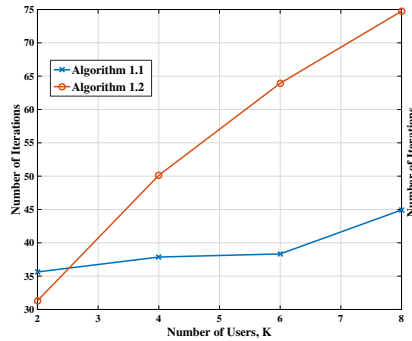

(a) Versus \# of users, $K$

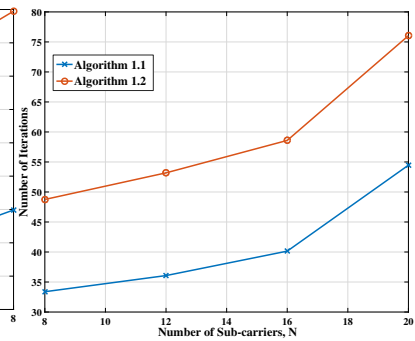

(b) Versus \# of sub-carriers, $N$ available carriers and required power drops significantly. Other than for $N=8$, i.e. $N=K$, under all levels of SIC error slice sub-carrier and rate reservations could be met. For $N=K$, the results are severely impacted by the limited feasibility of achieving the reservations for both slices. For $N=K$ and $\sigma_{e}^{2}=0.10$, no feasible solutions were found in this set of trials.

To examine the convergence and complexity of the proposed algorithms, the number of iterations for convergence of Algorithms 1.1 and 1.2 are plotted in Fig. 5 (a) for $N=16$, varying the number of users, and Fig. 5 (b) for $K=4$, varying the number of available sub-carriers, for $N^{\mathrm{rsv}}=1$ and $R^{\mathrm{rsv}}=1 \mathrm{bps} / \mathrm{Hz}$. As was noted in Section III-C, as $K$ and $N$ increase the required number of iterations for each algorithm to converge increases. The complexity of Algorithm 1.2 was found to be higher than that of Algorithm 1.1, which is confirmed in the required number of iterations depicted in Fig. 5 (a) and 5 (b). As expected, both algorithms are similarly impacted by increasing the number of subcarriers, $N$, and increasing the number of users, $K$, more significantly impacts Algorithm 1.2.

\section{CONClusion}

In this paper, we investigate the performance of uplink MC-NOMA in supporting VWNs in the presence of increased inter-user interference from errors in performing SIC. With the goal of minimizing required transmit power for battery dependent devices, while ensuring slice isolation and minimum system performance, we propose an iterative algorithm to solve the resulting non-convex and computationally intractable resource allocation problem. Via simulation results, the performance of the proposed algorithm is evaluated under both perfect and imperfect SIC constraints for rate and resource-based slice reservations. For comparison, results for OMA are also presented. We also investigate the relationship between SIC error, number of users and sub-carriers, and their combined effects on power requirements and resource utilization. Performance degrades with increasing levels of SIC error, as expected, however it is shown that the proposed algorithm for MC-NOMA outperforms OMA under all but the most extreme levels of SIC error. By minimizing required power, at high levels of inter-user interference multiplexing may not be favoured if slice reservations can be met at lower power with only a single user per sub-carrier, which is accounted for as a result of the optimization. Further, the results demonstrate that MC-NOMA can jointly meet slice reservations and support more users at lower transmit power than OMA.

\section{ACKNOWLEDGMENT}

This work is supported in part by an NSERC CRD Grant with Huawei Technologies Canada.

\section{REFERENCES}

[1] H. Tabassum, M. S. Ali, E. Hossain, M. J. Hossain, and D. I. Kim, "Non-orthogonal multiple access (NOMA) in cellular uplink and downlink: Challenges and enabling techniques," CoRR, vol. abs/1608.05783, 2016. [Online]. Available: http://arxiv.org/abs/1608.05783

[2] S. Lim and K. Ko, "Non-orthogonal multiple access (NOMA) to enhance capacity in 5G," Intl. Journal of Contents, vol. 11, no. 4, pp. 38-43, Dec 2015.

[3] M. Avriel and A. Williams, "Complementary geometric programming," SIAM Journal on Applied Mathematics, vol. 19, no. 1, pp. 125-141, Jul 1970.

[4] M. Chiang, C. W. Tan, D. Palomar, D. O'Neill, and D. Julian, "Power control by geometric programming," IEEE Trans. Wireless Commun., vol. 6, no. 7, pp. 2640-2651, Jul 2007.

[5] G. Xu, "Global optimization of signomial geometric programming problems," European Journal of Operational Research, vol. 233, no. 3, pp. 500-510, 2014.

[6] S. Boyd and L. Vandenberghe, Convex Optimization. Cambridge University Press, 2009. 